FACTA UNIVERSITATIS

Series: Physical Education and Sport, Vol. 17, No 1, 2019, pp. 149 - 163

https://doi.org/10.22190/FUPES180228016D

Research article

\title{
EFFECTS OF RULE CHANGES ON PERFORMANCE EFFICACY: DIFFERENCES BETWEEN WINNERS AND LOSERS TABLE TENNIS PLAYERS
}

\author{
UDC 796.386.01
}

\section{Zoran Đokić ${ }^{1}$, Gunter Straub ${ }^{2}$, Ivan Malagoli Lanzoni ${ }^{3}$, Michail Katsikadelis ${ }^{4}$, Goran Munivrana ${ }^{5}$}

\author{
${ }^{1}$ Faculty of Sport and Tourism, Educons University, Novi Sad, Serbia \\ ${ }^{2}$ Association of German Table Tennis Coaches (VDTT), Moers, Germany \\ ${ }^{3}$ School of Pharmacy, Biotechnology and Sport Science, University of Bologna, Italy \\ ${ }^{4}$ School of Physical Education \& Sport Science, Democritus University of Thrace, Komotini, Greece \\ ${ }^{5}$ Faculty of Kinesiology, University of Split, Split, Croatia
}

\begin{abstract}
The goal of this study was to analyze the differences in table tennis games between winning and losing players, considering changes of rules. The data taken from four major rule changes in 122 games between 244 players in the period 1996-2015 were analyzed. Performance indicators were 30 technical and tactical activities (type, total number, and stroke outcome) as well as serve and return activities (type, total number, serve outcome, and return outcome). A Mann-Whitney-Wilcoxon nonparametric test $(p \leq 0.05)$ was done. The results showed that the number of strokes per points considering change of rules varied, while domination of forehand play remained. The existence of statistically significant differences between winners and losers in all the analyzed periods: playing with a $38 \mathrm{~mm}$ ball to 21 points in eight activities, playing with a $40 \mathrm{~mm}$ ball till 21 points in one activity, playing with a $40 \mathrm{~mm}$ ball till 11 points in seven activities, and playing with new racket coverings in 14 activities were noted. The results showed the existence of different playing patterns, styles of play, and performance profiles for winning. Nowadays, players use, to a larger extent, different ways to win, but the effectiveness of the serve and return play gained importance.
\end{abstract}

Key Words: match analysis, individual sport, racket sport, performance indicators

Received February 28, 2018 / Accepted June 17, 2019

Corresponding author: Zoran Đokić

Educons University, Faculty of Sport and Tourism, Radnička 30a, 21000 Novi Sad 21000, Serbia

Phone: +381 21530231 •E-mail: zoran.djokic@tims.edu.rs 


\section{INTRODUCTION}

Table tennis is one of the most demanding games (Otcheva \& Drianovsky, 2002). Based on its structural complexity, table tennis as a sports game belongs to the poly-structural complex sports group dominated by open or semi-open movement structures that are performed in changing conditions (Ivanek, Đukić, Mikić, Smajić, \& Doder, 2018). It includes very fast adaptation to and decision-making in the tactics and techniques used. It is a complex sport with a large number of different types of strokes, even more specialized or individualized by the type of execution, the intensity, and the tactical goal (Tepper, 2003). To be successful, it is necessary that competitors have a highly developed mental capacity, physical fitness, as well as a technical and tactical performance capacity (Kondrić, FurjanMandić, Kondrić, \& Gabaglio, 2010). In high-level table tennis games, the number of competitions and played matches during the season increases from year to year. This situation leaves less time to undertake training in the competitive season and creates a need for increasing the efficiency of training (Đokić, 2007d). To stay the course with good results, more sports scientists are involved in providing support, covering all scientific disciplines, each focusing on improving the performance of the athlete (Hughes, Cooper, \& Nevill, 2004).

One segment of sport science support, with the main aim to develop and understand the game and help in the decision-making processes, is performance analysis (O'Donoghue, 2004; Padulo et al., 2016). Table tennis as a complex and dynamic sport requires observation and measurement to improve knowledge of performance, application of new knowledge to enhance performance, and an informed coaching process (Hughes et al., 2004).

Facing the problem of losing attractiveness, the International Table Tennis Federation (ITTF) introduced a series of reforms with a change in the rules: from October 2000, the diameter of the ball was increased from 38 to $40 \mathrm{~mm}$; from September 2001, a new playing system to 11 points was introduced; from September 2002, an unblock serve rule was introduced, and from September 2008, the equipment regulations were changed with regard to permissible racket coverings or rubbers. The covering material should be used as authorized by the ITTF, without any physical, chemical or other treatment, changing or modifying playing properties, friction, outlook, colour, structure, surface, etc. This rule change effectively bans speed gluing and the use of boosters or other chemicals applied to the sponge or the top rubber. There have been only a few studies that focused on performance analysis with change of rules (Wu \& Zhang, 2002; Đokić, 2002; Đokić, 2003; Li, Zhao, \& Zhang, 2005; Coupet \& Réache, 2007). Most of these studies confirmed that game structure more or less has been changed, but because of different descriptive procedures, and that the studies were done immediately upon the change of rules, full information about game change is lacking.

In racket sports, there are four types of performance analysis (PA): technique analysis, technical effectiveness, tactical analysis, and the physical aspects of performance. By doing this, two goals can be set: an improvement in the scientific understanding and help in sports practice sessions (McGarry, O'Donoghue, P., Sampaio, J., \& de Eira Sampaio, 2013). Physiological aspects have been verified in several studies (Đokić, 2004; Kondrić et al., 2010; Zagatto, Morel, \& Gobatto, 2010; 2016; Kondrić, Zagatto, \& Sekulić, 2013). Predominantly PA is concerned with tactical and technical evaluation and movement analysis (Carling, Reilly, \& Williams, 2008). This performance analysis data is very important because of their influence on the effectiveness of training sessions and competitions. As the performance analysts are gradually establishing their own methodological processes (Hughes \& Franks, 2007), there are several models applied in table tennis (Otcheva \& Drianovsky, 2002; Đokić, 2002, 2003b, 2007a, 2007b, 2007c; Hao, Cai, He, \& Hao, 2007; Wu \& Escobar Vargas, 
2007a, 2007b; Hao, Tian, Hao, \& Song, 2010; Katsikadelis, Pilianidis, \& Misichroni, 2010; Malagoli Lanzoni, Di Michele, R., \& Merni, 2011, 2012, 2014; Munivrana, Zekan Petrinović, \& Kondrić, 2015). In these analyses, authors were focused on technique analysis, technical effectiveness, as well as tactical and movement analyses. In these analyses, notational/match analysis dominates, which uses means to record aspects of individual performance. In historical terms, the oldest and still used model of table tennis analysis is the one of Đokic (2002) which provides more information than the others (Straub \& Klein-Soetebier, 2017).

We hypothesized that the structure in winning indicators in elite level table tennis competitions will be different after each major change of rules, and point out that game evolution over the years would modify the temporal structure.

The aim of this study is to compare the differences after the application of major changes of rules in the field of table tennis and especially in terms of performance indicators between winning and losing players.

\section{METHODS}

Data sample

The sample of participants consisted of 244 male table tennis players, in a total of 122 matches played from 1996 to 2015 at the following competitions: Olympic Games (2000 Sydney, 2004 Athens), World Championships (1995 Tjen Jin, 1997 Manchester, 1999 Eindhoven, 2001 Osaka, 2003 Paris, 2007 Zagreb, 2011 Rotterdam, 2014 Suzhou), European Championships (1998 Eindhoven, 2000 Bremen, 2002 Zagreb, 2003 Courmayer, 2007 Belgrade, 2009 Stuttgart, 2011 Gdansk), Commonwealth Games (2002 Manchester), ITTF Pro Tour tournaments (1996 Yugoslav Open, 1997 England Open, 1997 Austria Open, 1997 Sweden Open, 1998 French Open, 1998 Final Pro Tour - Hong Kong, 2001 Final Pro Tour Yokohama, 2003 Bulgaria Open), European League (1996 Yugoslavia vs Greece, 2002 Yugoslavia vs Sweden, 2003 Yugoslavia vs Austria), European Champions League (season 2000/2001), and German League (season 2000/2001). Four different periods in terms of rule changes were distinguished (Table 1).

Table 1 Four periods of rule changes in table tennis

\begin{tabular}{|c|c|c|c|c|}
\hline Period & Period label & $\begin{array}{l}\text { Time } \\
\text { frame }\end{array}$ & Innovation(s) & $\begin{array}{l}\text { Number } \\
\text { of cases } \\
\text { (matches) }\end{array}$ \\
\hline $\begin{array}{l}\text {... prior to the rule } \\
\text { changes starting with } \\
\text { the third millennium }\end{array}$ & "21-38mm" & $\begin{array}{c}1996- \\
09 / 2000\end{array}$ & & $n=30$ \\
\hline $\begin{array}{l}\ldots \text { of enlarging the } \\
\text { ball diameter }\end{array}$ & "21-40mm" & $\begin{array}{l}10 / 2000 \\
- \\
08 / 2001\end{array}$ & $\begin{array}{l}\text { Enlarging the ball diameter from } 38 \mathrm{~mm} \\
\text { to } 40 \mathrm{~mm} \text { while holding on to the old } \\
\text { scoring system to } 21 \text { points per game }\end{array}$ & $n=30$ \\
\hline $\begin{array}{l}\ldots \text { of implementing } \\
\text { a new scoring } \\
\text { system and new } \\
\text { service rule }\end{array}$ & "11-40mm" & $\begin{array}{l}09 / 2001 \\
- \\
08 / 2008\end{array}$ & $\begin{array}{l}\text { Shortening the game from } 21 \text { points to } \\
\text { only } 11 \text { (in addition, a new serve rule } \\
\text { from September } 2002 \text { onwards: The } \\
\text { serving behavior may not hide the ball } \\
\text { from the opponent) }\end{array}$ & $n=47$ \\
\hline $\begin{array}{l}\ldots \text { of new rubber } \\
\text { regulations }\end{array}$ & "new rub." & $\begin{array}{l}09 / 2008 \\
-2015 \\
\end{array}$ & $\begin{array}{l}\text { Ban on gluing rubbers freshly prior to a } \\
\text { match as well as using chemical boosters }\end{array}$ & $n=15$ \\
\hline
\end{tabular}


Players who preferred a distinct defensive style ("choppers", "chop-and-attack players") have been excluded from being a part of this sample, because the intention was to study the prevailing (offensive) playing style. All players were ranked in the top 100 in the ITTF Rank list.

Performance indicators

For the analysis of the games, technical and tactical parameters which are defined in the results between the winners and losers players were analysed (Đokić, 2002):

- Performed technical and tactical activities (TTA: total number of stroke and stroke outcomes; see Tepper (2003):

(1) FDRIV - forehand drive

(2) FBLOC - forehand block

(3) FFLIC - forehand flick

(4) FSPIN - forehand topspin - counter spin

(5) FSPBC - forehand topspin against backspin

(6) FPUSH - forehand push

(7) BDRIV - backhand drive

(8) BBLOC - backhand block

(9) BFLIC - backhand flick

(10) BSPIN - backhand topspin - counter spin

(11) BSPBC - backhand topspin against backspin

(12) BPUSH - backhand push

(13) ACEST - ace stroke (opponent did not make contact with the ball with his racket)

(14) WINST - winning stroke (opponent made error stroke)

(15) NORST - normal stroke (rally)

(16) ERROR - error stroke

(17) OVPLY - overplayed (situation after which opponent did not return to play or touch the ball)

- Performed serve and return of serve activities (SRA: efficacy of serve and serve outcomes, as well as return and return outcomes):

(18) FLONS - forehand long serve

(19) FSHOS - forehand short serve

(20) BLONS - backhand long serve

(21) BSHOS - backhand short serve

(22) SWDIR - point won with direct serve

(23) SWFIR - point won with the first stroke after serve

(24) SWACT - point won in action after serve

(25) SLOST - lost point after serve

(26) SEROR - serve error

(27) RWDIR - point won directly with return

(28) RWACT - point won in action after return

(29) RLOST - lost point after return

(30) REROR - return error 


\section{Procedure}

The data were collected by videos of matches, which had been recorded with digital cameras (Panasonic NV-DS28, Japan and Sony HDR-CX 190, Japan) and available via TV coverage on the official ITTF website. The video material allowed the observers during the video analysis to clearly see the players, the table, and the playing area, which allowed a reliable verification of all events during the match. The recordings were analysed in real speed, but in case of certain inconsistencies, they were re-winded and seen in slow motion $(0.2 \mathrm{X})$. All the data were registered in the specially prepared templates for the analysis of every match, in which all the analysed variables were coded and after that, the data were recorded in a Microsoft Excel spreadsheet.

\section{Reliability}

In order to ensure the quality of reliability (O'Donoghue \& Mayes, 2013), the matches were evaluated by means of intra-observers and inter-observers. For this research, two table tennis experts with adequate competition and coaching experience were engaged for the role of observers. The reliability of intra-observers is based on the concept of reanalysis of 20 random matches. The intra and inter-observers' reliability was evaluated by Krippendorff's Alpha. The reliability of the inter-observers was secured by reanalysis of all the matches by a second analyst. The intra-observers' reliability analysis showed an Alpha value of 0.993 .

\section{Statistics}

First, a descriptive analysis of the data was done. Second, a Mann-Whitney-Wilcoxon (MWW) nonparametric test was carried out with the goal of analysing performance indicators' differences between winning and losing players. The size of the impact (SI) was calculated by dividing the size $\mathrm{Z}$ with the square root of $\mathrm{N}$ (number of observations), that is using equation (1):

$$
r=\frac{\text { Zscore }}{(s q r t) N}
$$

The size of the impact was classified (Cohen, 1988, 1992) as small (from 0.1 to 0.3 ), medium ( 0.3 to 0.5$)$ or high (>0.5). All of the statistical analyses were done with a level of significance of $\mathrm{p} \leq 0.05$. All the data were analysed using SPSS 20.0 (IBM Corporation, USA).

\section{RESULTS}

\subsection{General analyses of game parameters}

Table 2 shows the forehand/backhand relations regarding stroke frequencies for all analysed players according to the analysed rules' periods as well as the average number of strokes in point (without serve).

The number of strokes per point contains the (regular) shots in accordance with the prevailing rules. A situation where a player touched the ball but did not successfully return it was not counted as a performed stroke. 
Table 2 Relation of forehand and backhand strokes (\%) and number of strokes per point

\begin{tabular}{lcccc}
\hline Period & $21-38 \mathrm{~mm}$ & $21-40 \mathrm{~mm}$ & $11-40 \mathrm{~mm}$ & New rub. \\
Year & $1996-2000$ & $2000-2001$ & $2001-2008$ & $2008-2015$ \\
\hline FH/BH* $(\%)$ & 58.242 .8 & $56.0 \quad 44.0$ & $58.0 \quad 42.0$ & 56.6 \\
Strokes per point & 2.12 & 2.08 & 2.43 & 2.16 \\
\hline
\end{tabular}

*FH - forehand strokes; $\mathrm{BH}$ - backhand strokes

The number of strokes per points considering different rules' periods varied, while forehand play prevailed. Table 3 shows the quality of the performed strokes (efficacy).

Table 3 Efficacy of performed strokes (\%)

\begin{tabular}{|c|c|c|c|c|c|}
\hline & ACEST & WINST & NORST & ERRO & VPLY \\
\hline $21-38 \mathrm{~mm}$ & 4,7 & 18,0 & 51,0 & 21,3 & 5,1 \\
\hline $21-40 \mathrm{~mm}$ & 4.7 & 19.3 & 47.7 & 23.0 & 5.3 \\
\hline $11-40 \mathrm{~mm}$ & 3.4 & 18.3 & 53.5 & 21.0 & 3.7 \\
\hline New rub. & 3.7 & 17.4 & 57.2 & 17.8 & 3.8 \\
\hline
\end{tabular}

With an enlargement of the ball diameter and the implementation of the new scoring system percentage of ace strokes, errors and situations where a competitor was overplayed decreased, while the number of normal strokes (rally) increased. In Table 4, the frequency (percentage) of stroke types is shown.

Table 4 Frequency of stroke types (\%)

\begin{tabular}{|c|c|c|c|c|c|c|c|c|c|c|c|c|}
\hline & FDRIV & $\overline{\mathrm{FBLO}}$ & $\overline{\text { FFLIC }}$ & $\overline{\text { FSPI }}$ & $\overline{\text { FSPB }}$ & $\overline{\text { FPUS }}$ & $\overline{\text { BDRIV }}$ & $\overline{B B L C}$ & BFLI & $\overline{B S P I N}$ & BSPB & BPUS \\
\hline $21-38 \mathrm{~mm}$ & 2.9 & 2.5 & 3.1 & 17 & 12.8 & 11.9 & 12.4 & 13.6 & 2.8 & 6.5 & 6.4 & 8.1 \\
\hline $21-40 \mathrm{~mm}$ & 0.9 & 3.3 & 5.5 & 19.9 & 12.2 & 14.1 & 7.5 & 11.1 & 4.2 & 8.8 & 5.7 & 6.8 \\
\hline $11-40 \mathrm{~mm}$ & 0.6 & 3.6 & 4.4 & 22.4 & 12.1 & 14.9 & 9.6 & 11.9 & 2.4 & 6.9 & 6.1 & 5.1 \\
\hline New rub. & 0.5 & 2.3 & 3.1 & 29.8 & 8.4 & 12.5 & 5.1 & 11.5 & 6.1 & 10.8 & 4.4 & 5.5 \\
\hline
\end{tabular}

According to the latest rule change(s), forehand and backhand offensive strokes as topspin against spin or block and backhand flick gained importance in use, while the use of passive strokes (drive, block) decreased. In addition, the use of topspin against backspin and the use of backhand push decreased in the course of time. Table 5 shows the efficacy of serve and return of serve play across the analysed periods of rule changes.

Table 5 Efficacy of serve and return of serve (\%)

\begin{tabular}{cccccccccc}
\hline & SWDIR & SWFIR & SWACT & SLOST & SEROR & RWDIR & RWACT & RLOST & REROR \\
\hline $21-38 \mathrm{~mm}$ & 14.4 & 24.5 & 18.5 & 40.7 & 1.9 & 18.4 & 24.4 & 42.5 & 14.6 \\
$21-40 \mathrm{~mm}$ & 16.7 & 25.0 & 14.8 & 40.7 & 2.8 & 19.7 & 22.9 & 40.9 & 16.5 \\
$11-40 \mathrm{~mm}$ & 13.1 & 22.7 & 17.9 & 44.0 & 2.3 & 17.5 & 27.4 & 41.0 & 14.0 \\
New rub. & 9.0 & 20.0 & 22.2 & 47.6 & 1.2 & 17.9 & 30.4 & 42.6 & 9.1 \\
\hline
\end{tabular}

With the shortening of the games and the unblock service rule, the efficacy in points directly won with serve and serve plus first stroke decreased while the number of points won in action after serve increased in the course of time. In essence, the number of lost 
points after the serve increased over time. In turn, it is to be noted that there are nowadays fewer errors made in regard to the return, and more points won in action after the serve receive.

\section{Performance efficacy differences between winners and losers}

Tables 6, 7, 8, and 9 show the results of analysis between match winners and losers in terms of the applied system of performance indicators considering the various rule change periods. The numbers indicate the arithmetic mean per played match regarding the winners and losers on a certain performance dimension, followed by the respective standard deviation, the results of the Mann-Whitney-Wilcoxon test, and the size of the impact.

Table 6 Performance indicators of winners and losers for the period 21-38mm

\begin{tabular}{|c|c|c|c|c|c|c|c|}
\hline \multirow[b]{2}{*}{ Variables } & \multirow{2}{*}{$\begin{array}{l}\text { Winners } \\
\text { Mean } \pm \text { SD }\end{array}$} & \multirow{2}{*}{$\begin{array}{c}\text { Losers } \\
\text { Mean } \pm \text { SD }\end{array}$} & \multicolumn{4}{|c|}{ MWW } & \multirow[b]{2}{*}{$\mathrm{p}$} \\
\hline & & & $\mathrm{U}$ & $\mathrm{W}$ & $\mathrm{Z}$ & SI & \\
\hline FDRIV & $6.0 \pm 4.0$ & $7.9 \pm 7.4$ & 422.0 & 887.0 & -.416 & & .677 \\
\hline FBLOC & $5.9 \pm 4.4$ & $6.2 \pm 4.5$ & 408.0 & 873.0 & -.625 & & .532 \\
\hline FFLIC & $6.8 \pm 6.9$ & $8.4 \pm 5.8$ & 341.5 & 806.5 & -1.608 & & .108 \\
\hline FSPIN & $43.6 \pm 20.4$ & $39.7 \pm 15.7$ & 431.5 & 896.5 & -.274 & & .784 \\
\hline FSPBC & $26.8 \pm 21.1$ & $36.1 \pm 19.6$ & 293.0 & 758.0 & -2.324 & 0.43 & $.020 *$ \\
\hline FPUSH & $33.2 \pm 15.2$ & $25.3 \pm 12.5$ & 309.5 & 774.5 & -2.080 & 0.38 & $.038 *$ \\
\hline BDRIV & $19.3 \pm 13.5$ & $24.6 \pm 15.1$ & 360.5 & 825.5 & -1.325 & & .185 \\
\hline BBLOC & $25.1 \pm 17.7$ & $22.8 \pm 12.2$ & 436.5 & 901.5 & -.200 & & .842 \\
\hline BFLIC & $4.7 \pm 5.2$ & $5.3 \pm 6.4$ & 444.0 & 909.0 & -.091 & & .928 \\
\hline BSPIN & $12.2 \pm 11.2$ & $10.6 \pm 10.9$ & 420.5 & 885.5 & -.437 & & .662 \\
\hline BSPBC & $8.7 \pm 5.4$ & $14.1 \pm 11.4$ & 343.5 & 808.5 & -1.580 & & .114 \\
\hline BPUSH & $12.7 \pm 7.7$ & $16.3 \pm 13.4$ & 398.0 & 863.0 & -.772 & & .440 \\
\hline ACEST & $10.8 \pm 4.9$ & $10.3 \pm 4.5$ & 431.0 & 896.0 & -.282 & & .778 \\
\hline WINST & $43.2 \pm 14.5$ & $37.3 \pm 12.4$ & 341.5 & 806.5 & -1.606 & & .108 \\
\hline NORST & $111.8 \pm 35.4$ & $116.8 \pm 31.9$ & 397.5 & 862.5 & -.777 & & .437 \\
\hline ERROR & $42.2 \pm 12.4$ & $53.3 \pm 14.6$ & 279.5 & 744.5 & -2.524 & 0.46 & $.012 *$ \\
\hline OVPLY & $11.2 \pm 4.0$ & $11.6 \pm 5.5$ & 435.5 & 900.5 & -.215 & & .830 \\
\hline FLONS & $17.3 \pm 13.6$ & $12.6 \pm 6.4$ & 416.5 & 881.5 & -.496 & & .620 \\
\hline FSHOS & $38.4 \pm 10.9$ & $46.4 \pm 15.3$ & 322.0 & 787.0 & -1.895 & & .058 \\
\hline BLONS & $.4 \pm .9$ & $.3 \pm .8$ & 419.0 & 884.0 & -.657 & & .511 \\
\hline BSHOS & $3.6 \pm 7.9$ & $1.1 \pm 2.7$ & 398.0 & 863.0 & -1.011 & & .312 \\
\hline SWDIR & $11.2 \pm 5.8$ & $6.4 \pm 2.9$ & 209.5 & 674.5 & -3.581 & 0.66 & $.000 * * *$ \\
\hline SWFIR & $15.4 \pm 5.5$ & $14.4 \pm 7.4$ & 377.5 & 842.5 & -1.075 & & .283 \\
\hline SWACT & $11.6 \pm 6.3$ & $10.8 \pm 4.4$ & 447.0 & 912.0 & -.045 & & .965 \\
\hline SLOST & $20.9 \pm 6.5$ & $28.4 \pm 7.4$ & 188.5 & 653.5 & -3.872 & 0.71 & $.000 * * *$ \\
\hline SEROR & $.8 \pm 1.0$ & $1.5 \pm 1.2$ & 287.0 & 752.0 & -2.523 & 0.46 & $.012 *$ \\
\hline RWDIR & $12.7 \pm 5.2$ & $9.1 \pm 3.7$ & 258.5 & 723.5 & -2.842 & 0.52 & $.004 * *$ \\
\hline RWACT & $15.1 \pm 3.6$ & $13.8 \pm 5.5$ & 374.0 & 839.0 & -1.127 & & .260 \\
\hline RLOST & $24.2 \pm 8.6$ & $26.1 \pm 8.3$ & 390.0 & 855.0 & -.889 & & .374 \\
\hline REROR & $6.7 \pm 3.3$ & $10.6 \pm 5.7$ & 250.0 & 715.0 & $-2, .973$ & 0.55 & $.003 * *$ \\
\hline
\end{tabular}

In the period of playing with the $38 \mathrm{~mm}$ ball to 21 points, eight performance indicators were significantly different between winners and losers. Three performance indicators were related to TTA and five were related to SRA. The statistically highly significant differences 
were found in lost point after serve $(\mathrm{z}=-3.872, \mathrm{p}<0.001, \mathrm{SI}=0.71)$, point won directly with serve $(z=-3.581, p<0.001, S I=0.66)$, also with high impact and error made in return of serve $(z=-2.973, p<0.01, S I=0.55)$, and point won directly with return $(z=-2.842, p<0.01$, $\mathrm{SI}=0.52)$. Then, there are statistically significant differences in made errors $(\mathrm{z}=-2.524, \mathrm{p}<$ $0.05, \mathrm{SI}=0.46)$ and serve errors $(\mathrm{z}=-2.523, \mathrm{p}<0.05, \mathrm{SI}=0.46)$. After that, statistically significant differences were found in forehand topspin on backspin $(\mathrm{z}=-2.324, \mathrm{p}<0.05$, $\mathrm{SI}=0.43)$ and forehand push $(\mathrm{z}=-2.080, \mathrm{p}<0.05, \mathrm{SI}=0.38)$.

Table 7 Performance indicators of winners and losers for the period 21-40mm

\begin{tabular}{|c|c|c|c|c|c|c|c|}
\hline \multirow[b]{2}{*}{ Variables } & Winners & Losers & \multicolumn{4}{|c|}{ MWW } & \multirow[b]{2}{*}{$\mathrm{p}$} \\
\hline & Mean \pm SD & Mean \pm SD & $\mathrm{U}$ & W & $\mathrm{Z}$ & SI & \\
\hline $\begin{array}{l}\text { FDRIV } \\
\end{array}$ & $1.2 \pm 1.3$ & $1.7 \pm 1.8$ & 392.0 & 888.0 & -.885 & & .376 \\
\hline FBLOC & $4.5 \pm 4.5$ & $5.6 \pm 5.6$ & 350.5 & 846.5 & -1.473 & & .141 \\
\hline FFLIC & $8.3 \pm 8.3$ & $8.4 \pm 6.5$ & 434.0 & 869.0 & -.230 & & .818 \\
\hline FSPIN & $29.9 \pm 9.7$ & $30.3 \pm 11.1$ & 447.0 & 943.0 & -.037 & & .970 \\
\hline FSPBC & $17.0 \pm 7.7$ & $20.1 \pm 5.8$ & 317.0 & 813.0 & -1.964 & 0.36 & $.050 *$ \\
\hline FPUSH & $22.7 \pm 8.8$ & $20.0 \pm 4.9$ & 371.5 & 806.5 & -1.156 & & .248 \\
\hline BDRIV & $11.9 \pm 8.6$ & $10.9 \pm 7.9$ & 411.5 & 846.5 & -.564 & & .573 \\
\hline BBLOC & $17.1 \pm 7.8$ & $16.1 \pm 6.7$ & 435.0 & 870.0 & -.215 & & .830 \\
\hline BFLIC & $6.7 \pm 5.0$ & $5.9 \pm 5.9$ & 396.0 & 831.0 & -.797 & & .426 \\
\hline BSPIN & $12.6 \pm 14.5$ & $14.0 \pm 12.3$ & 371.0 & 867.0 & -1.163 & & .245 \\
\hline BSPBC & $7.7 \pm 3.8$ & $9.6 \pm 6.3$ & 389.5 & 885.5 & -.890 & & .374 \\
\hline BPUSH & $10.3 \pm 7.6$ & $10.4 \pm 6.9$ & 427.5 & 923.5 & -.326 & & .744 \\
\hline ACEST & $7.4 \pm 3.8$ & $7.4 \pm 3.4$ & 366.5 & 801.5 & -1.231 & & .218 \\
\hline WINST & $31.3 \pm 8.8$ & $29.1 \pm 9.8$ & 426.0 & 922.0 & -.348 & & .728 \\
\hline NORST & $74.3 \pm 26.2$ & $75.5 \pm 26.9$ & 363.0 & 859.0 & -1.583 & & .113 \\
\hline ERROR & $34.3 \pm 8.9$ & $38.0 \pm 9.7$ & 372.0 & 868.0 & -1.258 & & .209 \\
\hline OVPLY & $8.2 \pm 5.1$ & $8.6 \pm 3.9$ & 434.5 & 930.5 & -.223 & & .824 \\
\hline FLONS & $8.5 \pm 4.9$ & $7.5 \pm 4.3$ & 374.0 & 809.0 & -1.118 & & .264 \\
\hline FSHOS & $34.3 \pm 10.2$ & $33.8 \pm 15.2$ & 434.0 & 930.0 & -.229 & & .819 \\
\hline BLONS & $.4 \pm 0.9$ & $.9 \pm 1.3$ & 338.0 & 834.0 & -1.651 & & .099 \\
\hline BSHOS & $2.1 \pm 4.7$ & $4.9 \pm 10.9$ & 413.5 & 909.5 & -.535 & & .593 \\
\hline SWDIR & $7.9 \pm 3.9$ & $7.9 \pm 4.6$ & 440.5 & 875.5 & -.134 & & .894 \\
\hline SWFIR & $12.6 \pm 4.2$ & $11.2 \pm 4.1$ & 353.0 & 788.0 & -1.431 & & .152 \\
\hline SWACT & $7.0 \pm 3.9$ & $7.1 \pm 4.8$ & 449.0 & 884.0 & -.007 & & .994 \\
\hline SLOST & $18.3 \pm 5.3$ & $20.5 \pm 5.9$ & 338.0 & 834.0 & -1.652 & & .098 \\
\hline SEROR & $.9 \pm 1.3$ & $1.8 \pm 1.7$ & 330.5 & 826.5 & -1.850 & & .064 \\
\hline RWDIR & $8.4 \pm 3.6$ & $9.4 \pm 4.8$ & 405.5 & 901.5 & -0.654 & & .513 \\
\hline RWACT & $11.1 \pm 4.2$ & $9.6 \pm 4.2$ & 347.0 & 782.0 & -1.522 & & .128 \\
\hline RLOST & $17.8 \pm 6.3$ & $19.2 \pm 5.9$ & 391.5 & 887.5 & -0.860 & & .390 \\
\hline REROR & $6.6 \pm 4.2$ & $8.3 \pm 4.3$ & 333.5 & 829.5 & -1.723 & & .085 \\
\hline
\end{tabular}

In the period of playing with the $40 \mathrm{~mm}$ ball to 21 points, only one performance indicator was significantly different between winners and losers. It was related to TTA the use of forehand topspin on backspin $(\mathrm{z}=-1.964, \mathrm{p} \leq 0.05)$ with medium impact $(\mathrm{SI}=0.36)$. 
Table 8 Performance indicators of winners and losers for the period 11-40mm

\begin{tabular}{|c|c|c|c|c|c|c|c|}
\hline \multirow[b]{2}{*}{ Variables } & \multirow{2}{*}{$\begin{array}{c}\text { Winners } \\
\text { Mean } \pm \text { SD }\end{array}$} & \multirow{2}{*}{$\begin{array}{c}\text { Losers } \\
\text { Mean } \pm \text { SD }\end{array}$} & \multicolumn{4}{|c|}{ MWW } & \multirow[b]{2}{*}{$\mathrm{p}$} \\
\hline & & & $\mathrm{U}$ & $\mathrm{W}$ & $\mathrm{Z}$ & SI & \\
\hline FDRIV & $2.3 \pm 4,9$ & $2.4 \pm 4.2$ & 606.5 & 1236.5 & -.073 & & .942 \\
\hline FBLOC & $4.9 \pm 4.1$ & $5.8 \pm 3.2$ & 469.0 & 1099.0 & -1.698 & & .090 \\
\hline FFLIC & $7.4 \pm 5.5$ & $5.9 \pm 5.1$ & 508.5 & 1138.5 & -1.226 & & .220 \\
\hline FSPIN & $34.5 \pm 15.8$ & $32.6 \pm 14.2$ & 575.5 & 1205.5 & -.435 & & .664 \\
\hline FSPBC & $17.3 \pm 9.5$ & $16.1 \pm 8.3$ & 593.0 & 1223.0 & -.229 & & 819 \\
\hline FPUSH & $23.3 \pm 11.9$ & $21.3 \pm 14.1$ & 551.5 & 1181.5 & -.717 & & .473 \\
\hline BDRIV & $13.2 \pm 14.4$ & $15.7 \pm 15.1$ & 529.5 & 1159.5 & -.977 & & .329 \\
\hline BBLOC & $16.6 \pm 8.3$ & $18.9 \pm 11.3$ & 553.5 & 1183.5 & -.694 & & .488 \\
\hline BFLIC & $3.1 \pm 3.8$ & $3.9 \pm 4.2$ & 564.0 & 1194.0 & -.579 & & .563 \\
\hline BSPIN & $9.5 \pm 7.4$ & $11.1 \pm 7.5$ & 533.0 & 1163.0 & -.936 & & .350 \\
\hline BSPBC & $8.2 \pm 6.4$ & $10.2 \pm 7.9$ & 539.0 & 1169.0 & -.865 & & .387 \\
\hline BPUSH & $7.0 \pm 7.3$ & $8.2 \pm 6.9$ & 525.5 & 1155.5 & -1.025 & & .305 \\
\hline ACEST & $5.7 \pm 3.0$ & $4.9 \pm 1.9$ & 537.0 & 1167.0 & -.894 & & .371 \\
\hline WINST & $32.1 \pm 8.8$ & $25.0 \pm 8.6$ & 339.5 & 969.5 & -3.210 & 0.47 & $.001 * *$ \\
\hline NORST & $81.7 \pm 28.1$ & $84.9 \pm 28.7$ & 565.0 & 1195.0 & -.558 & & .577 \\
\hline ERROR & $29.1 \pm 12.3$ & $36.3 \pm 8.9$ & 358.0 & 988.0 & -2.992 & 0.44 & $.003 * *$ \\
\hline OVPLY & $5.3 \pm 2.3$ & $6.3 \pm 3.6$ & 534.0 & 1164.0 & -.930 & & .353 \\
\hline FLONS & $20.3 \pm 14.7$ & $18.9 \pm 15.2$ & 440.0 & 905.0 & -.148 & & .882 \\
\hline FSHOS & $37.5 \pm 13.6$ & $38.4 \pm 23.1$ & 432.5 & 897.5 & -.259 & & .795 \\
\hline BLONS & $.5 \pm .9$ & $.5 \pm 1.0$ & 435.0 & 900.0 & -.292 & & .770 \\
\hline BSHOS & $3.9 \pm 7.6$ & $2.7 \pm 3.9$ & 417.0 & 882.0 & -.565 & & .572 \\
\hline SWDIR & $5.4 \pm 3.4$ & $5.1 \pm 2.9$ & 602.0 & 1232.0 & -.124 & & .901 \\
\hline SWFIR & $9.6 \pm 4.3$ & $8.6 \pm 3.8$ & 535.0 & 1165.0 & -.916 & & .360 \\
\hline SWACT & $8.7 \pm 3.9$ & $5.8 \pm 3.2$ & 369.0 & 999.0 & $-2.873 * *$ & 0.42 & $.004 * *$ \\
\hline SLOST & $15.7 \pm 4.6$ & $19.6 \pm 3.9$ & 331.5 & 961.5 & $-3.308 * *$ & 0.48 & $.001 * *$ \\
\hline SEROR & $.9 \pm .9$ & $.9 \pm .9$ & 586.5 & 1216.5 & -.327 & & .744 \\
\hline RWDIR & $7.7 \pm 3.1$ & $6.2 \pm 2.9$ & 429.5 & 1059.5 & $-2.164 *$ & 0.32 & $.030 *$ \\
\hline RWACT & $12.1 \pm 4.7$ & $9.5 \pm 3.5$ & 419.5 & 1049.5 & $-2.275^{*}$ & 0.33 & $.023 *$ \\
\hline RLOST & $14.5 \pm 5.8$ & $17,9 \pm 6.1$ & 398.5 & 1028.5 & $-2.520^{*}$ & 0.37 & $.012 *$ \\
\hline REROR & $5.6 \pm 3.2$ & $5.5 \pm 3.6$ & 587.0 & 1217.0 & -.302 & & .762 \\
\hline
\end{tabular}

In the period of playing with the $40 \mathrm{~mm}$ ball to 11 points and unblock serve, seven performance indicators were significantly different between winners and losers. Two performance indicators were related to TTA and five were related to SRA. The statistically highly significant differences were found in lost point on service $(\mathrm{z}=-3.308, \mathrm{p}$ $<0.01)$, winning stroke $(\mathrm{z}=-3.210, \mathrm{p}<0.01)$, made errors $(\mathrm{z}=-2.992, \mathrm{p}<0.01)$, point won after serve $(\mathrm{z}=-2.873, \mathrm{p}<0.01)$, lost point after receive of serve $(\mathrm{z}=-2.520, \mathrm{p}<0.05)$, point won after serve receive $(z=-2.275, p<0.05)$. Statistically significant differences were also found in points won directly with serve receive $(z=-2.164, p<0.05)$. Impact size in all differences was medium ( $\mathrm{SI}=0.32-0.48)$. 
Table 9 Performance indicators of winners and losers for the period new rub.

\begin{tabular}{|c|c|c|c|c|c|c|c|}
\hline \multirow[b]{2}{*}{ Variables } & \multirow{2}{*}{$\begin{array}{c}\text { Winners } \\
\text { Mean } \pm \text { SD }\end{array}$} & \multirow{2}{*}{$\begin{array}{c}\text { Losers } \\
\text { Mean } \pm \text { SD }\end{array}$} & \multicolumn{4}{|c|}{ MWW } & \multirow[b]{2}{*}{$\mathrm{p}$} \\
\hline & & & $\mathrm{U}$ & W & $\mathrm{Z}$ & SI & \\
\hline FDRIV & $.4 \pm 1.1$ & $.7 \pm 1.8$ & 104.0 & 224.0 & -.543 & & .587 \\
\hline FBLOC & $2.9 \pm 3.6$ & $2.9 \pm 2.6$ & 95.5 & 215.5 & -.716 & & .474 \\
\hline FFLIC & $2.8 \pm 2.3$ & $5.0 \pm 3.0$ & 69.5 & 189.5 & -1.798 & & .072 \\
\hline FSPIN & $37.9 \pm 17.6$ & $36.8 \pm 13.3$ & 110.0 & 230.0 & -.104 & & .917 \\
\hline FSPBC & $13.6 \pm 6.6$ & $7.5 \pm 3.8$ & 41.0 & 161.0 & -2.978 & 0.77 & $.003 * *$ \\
\hline FPUSH & $17.9 \pm 7.7$ & $13.5 \pm 7.4$ & 65.0 & 185.0 & -1.975 & 0.51 & $.048 *$ \\
\hline BDRIV & $6.6 \pm 4.5$ & $6.1 \pm 7.3$ & 87.5 & 207.5 & -1.045 & & .296 \\
\hline BBLOC & $11.3 \pm 5.5$ & $17.5 \pm 10.2$ & 61.5 & 181.5 & -2.127 & 0.55 & $.033 *$ \\
\hline BFLIC & $5.4 \pm 3.5$ & $10.0 \pm 7.6$ & 73.0 & 193.0 & -1.651 & & .099 \\
\hline BSPIN & $14.7 \pm 11.7$ & $12.4 \pm 7.3$ & 105.5 & 225.5 & -.291 & & .771 \\
\hline BSPBC & $3.6 \pm 2.8$ & $7.5 \pm 4.2$ & 44.0 & 164.0 & -2.861 & 0.74 & $.004 * *$ \\
\hline BPUSH & $5.9 \pm 5.6$ & $7.8 \pm 8.4$ & 109.0 & 229.0 & -.146 & & .884 \\
\hline ACEST & $5.1 \pm 1.7$ & $4.0 \pm 2.1$ & 74.5 & 194.5 & -1.616 & & .106 \\
\hline WINST & $26.6 \pm 5.3$ & $15.7 \pm 9.3$ & 36.0 & 156.0 & -3.195 & 0.83 & $.001 * *$ \\
\hline NORST & $71.4 \pm 28.1$ & $67.5 \pm 32.6$ & 102.5 & 222.5 & -.415 & & .678 \\
\hline ERROR & $17.7 \pm 7.3$ & $25.7 \pm 9.5$ & 58.0 & 178.0 & -2.264 & 0.58 & $.024 *$ \\
\hline OVPLY & $3.6 \pm 2.2$ & $5.7 \pm 1.3$ & 42.0 & 162.5 & -2.955 & 0.76 & $.003 * *$ \\
\hline FLONS & $3.9 \pm 1.9$ & $5.1 \pm 3.9$ & 98.0 & 218.0 & -.608 & & .543 \\
\hline FSHOS & $24.9 \pm 7.4$ & $20.0 \pm 9.8$ & 76.0 & 196.0 & -1.521 & & .128 \\
\hline BLONS & $.4 \pm 1.6$ & $.7 \pm 1.5$ & 99.0 & 219.0 & -.948 & & .343 \\
\hline BSHOS & $.7 \pm 2.8$ & $3.5 \pm 7.7$ & 96.0 & 216.0 & -1.158 & & .247 \\
\hline SWDIR & $3.9 \pm 2.4$ & $1.5 \pm 1.2$ & 48.0 & 168.0 & -2.727 & 0.70 & $.006 * *$ \\
\hline SWFIR & $7.6 \pm 2.7$ & $4.5 \pm 3.2$ & 54.5 & 174.5 & -2.424 & 0.63 & $.015 *$ \\
\hline SWACT & $7.3 \pm 3.4$ & $6.1 \pm 2.2$ & 93.5 & 213.5 & -.799 & & .424 \\
\hline SLOST & $11.3 \pm 4.4$ & $17.3 \pm 2.6$ & 27.5 & 147.5 & -3.545 & 0.92 & $.000^{* * * *}$ \\
\hline SEROR & $.2 \pm .4$ & $.5 \pm .7$ & 87.0 & 207.0 & -1.317 & & .188 \\
\hline RWDIR & $6.5 \pm 2.6$ & $4.1 \pm 3.1$ & 58.5 & 178.5 & -2.267 & 0.59 & $.023 *$ \\
\hline RWACT & $10.9 \pm 3.1$ & $7.1 \pm 2.8$ & 39.5 & 159.5 & -3.055 & 0.79 & $.002 * *$ \\
\hline RLOST & $10.6 \pm 4.2$ & $14.5 \pm 2.8$ & 55.5 & 175.5 & -2.391 & 0.62 & $.017 *$ \\
\hline REROR & $1.6 \pm 1.3$ & $3.8 \pm 2.6$ & 51.0 & 171.0 & -2.601 & 0.67 & $.009 *$ \\
\hline
\end{tabular}

In the period of playing with new rubbers, 14 performance indicators were significantly different between winners and losers. Seven performance indicators were related to TTA and seven related to SRA. Statistically highly significant differences were found in lost point after serve $(\mathrm{z}=-3.545, \mathrm{p}<0.001)$, winning stroke $(\mathrm{z}=-3.195, \mathrm{p}<0.01)$, while the statistically significant differences were found in points won after serve receive $(\mathrm{z}=-3.055, \mathrm{p}$ $<0.01)$, playing forehand topspin on backspin $(\mathrm{z}=-2.978, \mathrm{p}<0.01)$, been in an overplayed situation $(\mathrm{z}=-2.955, \mathrm{p}<0.01)$, playing backhand topspin on backspin $(\mathrm{z}=-2.861, \mathrm{p}<0,01)$, points won directly with a serve $(\mathrm{z}=-2.727, \mathrm{p}<0.01)$, errors in return serve $(\mathrm{z}=-2.601, \mathrm{p}<$ $0.01)$, points won with the first stroke after serve $(z=-2.424, p<0.05)$, lost points after serve receive $(\mathrm{z}=-2.391, \mathrm{p}<0.05)$, points won directly in serve receive $(\mathrm{z}=-2.267, \mathrm{p}<0.05)$, made errors $(\mathrm{z}=-2.264, \mathrm{p}<0.05)$, playing backhand block $(\mathrm{z}=-2.127, \mathrm{p}<0.05)$, and forehand push $(\mathrm{z}=-1.975, \mathrm{p}<0.05)$. The impact size in all the differences was high $(\mathrm{SI}=0.51-0.92)$. 


\section{DISCUSSION}

The purpose of this study was to analyze the differences caused in the game due to the changes in the rules in general and between winning and losing players in particular. The results from the present study indicate that changes in the rules influence a change of playing patterns between winning and losing performances.

The findings of this study are that, considering the changes in the rules, the number of strokes per point was variable with the tendency of the game to be more fast and dynamic with shortening point rallies as the studies of Otcheva \& Drianovski (2002) and Li et al. (2005) already claimed. This includes a decreasing use of passive forehand strokes, which were used in conditions of extreme difficulty (Malagoli Lanzoni et al., 2014), while the domination of forehand offensive play prevailed and got greater importance as Zhang \& Hohmann (2004) and Malagoli Lanzoni et al. (2011) concluded, too.

Increasing the diameter of the ball reduced the speed of the ball and the spin $(\mathrm{Wu} \&$ Zhang, 2002), and this possibly had an influence on the number of ace strokes which are related to a decrease in overplay situations, while the number of normal strokes in a rally increased. It is very interesting that increasing the diameter of the ball alone did not result in an apparent prolongation of the rally. This effect did not occur till the shortening of the games a year later (2001). This prolongation of the rally could have been due to the circumstance where the players were forced to heighten their level of attention, and also, it might have been the consequence of the fact that the new ball could be handled much easier by the players after a year of gathering experience. Enlarging the ball diameter, shortening the games, establishing more transparency in regard to the serve, and new requirements in terms of equipment might have especially influenced the efficacy of the serve-and-return play. In the course of time, the impact of the serve diminished, which, in reverse, offered better chances to the receiver to win points when the opponent had to serve.

In the period before changing the rules, the results of analyses revealed eight distinguishing performance indicators. Performance indicators were more related to serve and return of serve activities than technical and tactical activities in the long run of the rallies. Results suggest that winning players used more forehand push as neutral strokes, frequently used at the beginning of the rally with a high proportion of serve returns (Malagoli Lanzoni et al., 2014). On the other hand, the technique of pushing the ball must not be seen as a neutral stroke because good players can push very effectively in terms of variations of spin and placement as well as feigning movements, provoking subpar attacks on the side of the opponent. Players who lost the match, compared to this, seemed to try more intensively to attack first using forehand topspin against a serve or a push of the opponent. At first glance, this appears to be a good choice, but this stroke has to have a high quality (in respect of spin, placement, and flying height of the ball), so that the opponent cannot counterattack easily or effectively. Thus, a forehand topspin against a backspin can have a rather negative outcome when it is performed badly. Analyzing the playing patterns in the period prior to the diverse rule changes also shows that match winners are much better in regard to the realization of points won directly both with the serve and the serve return.

With the enlargement of the ball in the old playing system to 21 points, which lasted only one season, analyses pointed to the existence of only one performance indicator between winners and losers. The general lack of significant differences for the analyzed games in this period suggests the existence of different playing patterns, styles of play, performance profiles, and makes one suppose that the results of play were more accidental 
(Wu \& Zhang, 2002). We can assume that this was a period of adaptation, orientation, and experimentation, which lasted only one year of play and the analyzed matches took place in the middle of the season. The significant performance indicator was related to TTA. Again, it can be noted that losing players tried to use the forehand topspin against backspin more often.

With the shortening of the game (the new scoring system) and the unblock service rule, analyses revealed seven distinguishing performance indicators, of which two performance indicators were related to TTA (the efficacy of the performed strokes) and five were related to SRA. It seems, indeed, like Dominicy, Ley, \& Swan (2013) claimed, that the new scoring system quickly equalizes both players by increasing the potential number of crucial points without influencing the relative strengths of the players too much. The findings suggest that good control and well-placed strokes brought an advantage to winning players, who made fewer errors and more winning strokes than losing players. In regard to SRA, there were two performance indicators related with the realization of one's own serve and three with the efficacy in returning the opponent's serve. As Coupet \& Réache (2007) concluded, the reduced serve numbers in the course of shortened games decreases the dominance between the players in terms of directly won points with a serve. Regarding entire rallies, however, event results suggest that winners have better realization in action after their own serve, which is the opposite situation compared to the period before implementing the rule changes. The findings draw attention to the fact that winners are also superior in respect to receiving the opponent's serve. Results likely implicate that performance indicators related to receive gain importance and prevail.

In the period of introduction of the new rubbers rule, analyses revealed differences with respect to 14 distinguishing performance indicators, of which seven were related to TTA and seven related to SRA. Winning players try to focus even more on a forehand-oriented style of play right from the beginning of a rally. In contrast to the two periods, when the old scoring system was in use ("21-38mm", " $21-40 \mathrm{~mm}$ "), now the winners tend to intensively start attacking with a forehand topspin on backspin, which is regarded as one of the most aggressive strokes (Malagoli Lanzoni et al., 2014). Besides now, as in the period prior to the rule inventions, winners relatively use more forehand push as a neutral stroke, mostly in terms of a serve receive. By contrast, as an answer to a push or serve, their opponents were more frequently in situations to play backhand topspin on backspin and backhand block, which are strokes that tend to have a negative outcome (Malagoli Lanzoni et al., 2014). Both facts taken together create the idea that successful players nowadays may especially be good at starting their forehand topspin play from the backhand side of the table.

In terms of SRA, there were three activities related with the realization of the own serve and four with efficacy in returning the opponent's serve. Compared to all former periods of rule changes, it seems likely that new rubbers allow both serve and serve return and has become even more, the primary distinguishing feature between winner and losers. Thus, in the points accumulated by the winning player, the points won directly and with the first stroke after serve, as well as points won directly in return of serve prevailed. A recent case analysis (Zhang, Liu, Hu, \& Liu, 2013) indicated a correlation between technique effectiveness and competition performance based exactly on this point, which makes a difference between winners and losers. In this current study, significant differences in terms of performance indicators related to serve receive were seen, namely on all four examined return-related dimensions. Thus, the somewhat classic quote by Muster (1999, p. 254): "The return to a serve is the most important shot in table tennis," can be emphasized at this juncture anew. 
Consequently, the aim of this study was to determine which performance indicators were related to a player's success. The results should help in maximizing the effectiveness of practice time, considering that this is the most important aspect of the game which makes a difference regarding the match outcome and quality, by throwing light on many factors that can disrupt training sessions.

\section{CONCLUSION}

The results of the present study indicate that the rule changes made in table tennis from the year 2000 to 2008 might indeed have contributed extensively to a change of playing patterns. In particular, the repeated changes within the set of rules could have been a vital cause for partially defusing the impact of the serve over the course of one and a half decades. Nevertheless, the quality of serve-and-return play is more obviously than ever before the primary differentiator between winners and losers in table tennis.

The results of this research can be useful for coaches and players with regard to the identification of important aspects of the game, in order to design better training sessions. Also, the findings can be seen as a walking advertisement for a particular model of performance analysis indicating a need for continuous systematic match observation in this sport. This may be even more true when looking into the future, bearing in mind the implementation of two types of plastic balls throughout the last two seasons.

\section{REFERENCES}

Carling, C., Reilly, T., \& Williams, A.M. (2008). Performance Assessment for Field Sports. London, UK: Routledge.

Cohen, J.W. (1988). Statistical power analysis for the behavioral sciences ( $2^{\text {nd }}$ Edition). Hillsdale, NJ: Lawrence Erlbaum Associates.

Cohen, J.W. (1992). Quantitative methods in psychology: A power primer. Psychological Bulletin, 112, 155-159.

Coupet, A., \& Réache, L.G. (2007). Comparison between table tennis scoring system (11 \& 21 points) by probabilistic simulation. In X.P. Zhang, D.D. Xiao, \& Y. Dong (Eds.), The Proceedings of the Ninth International Table Tennis Federation Sports Science Congress $2^{\text {nd }}$ Edition. Paper presented at the 9th ITTF Sports Science Congress, Shanghai, China, 27-30 April 2005 (pp. 35-40). Beijing: People's Sport Publishing House of China.

Đokić, Z. (2002). Structure of competitors activities of top table tennis players. International Journal of Table Tennis Sciences, 4-5, 74-90.

Đokić, Z. (2003). Service and service return in modern top table tennis. Programme and abstracts. Paper presented at the $8^{\text {th }}$ International Table Tennis Federation Sports Science Congress-The $3^{\text {rd }}$ World Congress of Science and Racket Sports, Paris, France, 17-19 May (pp. 21). Paris: INSEP.

Đokić, Z. (2004). Heart rate monitoring of table tennis players. In A. Lees, J. F. Kahn \& I. W. Maynard (Eds.), Science and Racket Sports III (pp. 21-22). London and New York: Routledge.

Đokić, Z. (2005). Changing rules in table tennis. In N. Dikić, S. Živanić, S. Ostojić, \& Z. Tornjanski (Eds.), Abstract Book. Paper presented at the $10^{\text {th }}$ Annual Congress of the European College of Sport Science, Belgrade, 13-16 July (pp. 352). Belgrade: Sports Medicine Association of Serbia.

Đokić, Z. (2007a). The use of forehand spin in modern table tennis. In X.P. Zhang, D.D. Xiao, \& Y. Dong (Eds.), The proceedings of the Ninth International Table Tennis Federation Sports Science Congress $2^{\text {nd }}$ edition. Paper presented at the 9th ITTF Sports Science Congress, Shanghai, China, 27-30 April 2005 (pp.152-158). Beijing: People's Sport Publishing House of China.

Đokić, Z. (2007b). Differences in tactics in game of top table tennis players and others-factor of success in top table tennis. In X.P. Zhang, D.D. Xiao, \& Y. Dong (Eds.), The proceedings of the Ninth International Table Tennis Federation Sports Science Congress $2^{\text {nd }}$ Edition. Paper presented at the 9th ITTF Sports Science Congress, Shanghai, China, 27-30 April 2005 (pp. 138-145). Beijing: People's Sport Publishing House of China. 
Đokić, Z. (2007c). Backhand game in top table tennis. In X.P. Zhang, D.D. Xiao, \& Y. Dong (Eds.), The proceedings of the Ninth International Table Tennis Federation Sports Science Congress $2^{\text {nd }}$ Edition. Paper presented at the 9th ITTF Sports Science Congress, Shanghai, China, 27-30 April 2005 (pp. 145-152). Beijing: People's Sport Publishing House of China.

Đokić, Z. (2007d). Functional diagnostics of top table tennis players. In M. Kondrič, \& G. Furjan Mandić (Eds.), Proceedings book of the 10th Anniversary ITTF Sports Science Congress (pp. 168-174). Zagreb: University of Zagreb, Faculty of kinesiology; Croatian Table Tennis Association; International Table Tennis Federation.

Dominicy, Y., Ley, C., \& Swan, Y. (2013). A stochastic analysis of table tennis, Brazilian Journal of Probability and Statistics, 27(4), 467-486.

Hao, Z., Tian, Z., Hao, Y., \& Song, J. (2010). Analysis on technique and tactics of Lin Ma and Hao Wang in the men's single table tennis final in the $29^{\text {th }}$ Olympic Games, International Journal of Table Tennis Sciences, 6 , 74-78.

Hao, Z., Cai, X.L., He, F., \& Hao, Y.J. (2007). Analysis on technique and tactics of RyuSeung-Min in men's singles table tennis final and semifinal of the $28^{\text {th }}$ Olympic Games in Athens. In M. Kondrić, \& G. FurjanMandić (Eds.), The Proceedings of the $10^{\text {th }}$ Anniversary International Table Tennis Federation Sport Science Congress (pp. 374-382). Paper presented at the $10^{\text {th }}$ ITTF Sport Science Congress, Zagreb, Croatia, 18-20 May. Zagreb, Lausanne: ITTF.

Hughes, M., Cooper, S.M. \& Nevill, A. (2004). Analysis of notation data: reliability. In Hughes, M. \& Franks, M.I. (Eds.) Notational Analysis of Sport-Systems for better coaching and performance in sport (pp.189 - 204). Second Edition, London: Routledge.

Hughes, M., \& Franks, I. (2007). The essentials of performance analysis: an introduction. Routledge.

Ivanek, V., Đukić, B., Mikić, B., Smajić, M., \& Doder, D. (2018). Effects of technical and tactical characteristics on the performance of the table tennis players. Facta Universitatis Series Physical Education and Sport, 16(1), $157-166$

Katsikadelis, M., Pilianidis, T., \& Misichroni, A. (2010). Comparison of Rally Time in XXIX Beijing (2008) and XXVIII Athens (2004) Olympic Table Tennis Tournaments. International Journal of Table Tennis Sciences, 6 , 55-59.

Kondrić, M., Furjan-Mandić, G., Kondrić, L., \& Gabaglio, A. (2010). Physiological demands and testing in table tennis. International Journal of Table Tennis Sciences, 6, 165-170.

Kondrić, M., Zagatto, A.M., \& Sekulić, D. (2013). The physiological demands of table tennis: A review. Journal of Sports Science and Medicine, 12(3), 362-370.

Li, J.L., Zhao, X., \& Zhang, C.H. (2005). Changes and development: Influence of new rules on table tennis techniques. International Journal of Table Tennis Sciences, 11, 67-72.

Malagoli-Lanzoni, I., Di Michele, R., \& Merni, F. (2011). Performance indicators in table tennis: a review of the literature. International Journal of Table Tennis Sciences, 7, 71-75.

Malagoli Lanzoni, I., Di Michele, R., \& Merni F. (2012). Reliability of selected performance indicators in table tennis, International Journal of Table Tennis Sciences, 7, 62- 65.

Malagoli Lanzoni, I., Di Michele, R., \& Merni F. (2014). A notational analysis of shot characteristics in top-level table tennis players, European Journal of Sport Science, 14(4), 309-317.

McGarry, T., O'Donoghue, P., Sampaio, J., \& de Eira Sampaio, A.J. (2013). Routledge handbook of sports performance analysis. Routledge.

Munivrana, G., Zekan Petrinović, L., \& Kondrić, M. (2015). Structural analysis of technical-tactical elements in table tennis and their role in different playing zones. Journal of Human Kinetics, 47, 197-214.

Muster, M. (1999). Zur Bedeutung des "situativen Trainings" im Hochleistungstischtennis - empirische Untersuchung zur Identifikation von "Spielsituationen" (The significance of "situational training" in highperformance table tennis - an empirical study for the identification of "game-play situations"). Unpublished doctoral dissertation. Technical University of Munich, Munch, Germany. In German

O'Donoghue, P. (2004). Match analysis in racket sports. In A. Lees, J.F. Kahn \& I.W. Maynard (Eds.), Science and Racket Sports III (pp. 155-162). London: Routledge.

O'Donoghue, P. \& Mayes, A. (2013), Performance analysis, feedback and communication in coaching. In T. McGarry, P. O' Donoghue, \& J. Sampaio (Eds.) Routledge Handbook of Sport Performance Analysis (pp.155164). Routledge.

Otcheva, G., \& Drianovsky, Y. (2002). Comparative analysis of the games of the finalists from the biggest international and Bulgarian table tennis competition 2000. Table Tennis Sciences, 5, 155-166.

Padulo, J., Pizzolato, F., Tosi Rodrigues, S., Migliaccio, G.M., Attene, G., Curcio, R., et al. (2016). Task complexity reveals expertise of table tennis players. J Sports Med Phys Fitness. 56(1-2), 149-156.

Straub, G., \& Klein-Soetebier, T. (2017). Analytic and descriptive approaches to systematic match analysis in table tennis. German Journal of Exercise and Sport Research, 47(2), 95-102.

Tepper, G. (2003). ITTF Level 1 coaching manual. Shanghai Minsun Packaging \& Printing Company: ITTF. 
Zagatto, A.M., Morel, E.A., \& Gobatto, C.A. (2010). Physiological responses and characteristics of table tennis matches determined in official tournaments, Journal of Strength and Conditioning Research, 24(4), 942-949.

Zhang, H., \& Hohmann, A. (2004). Performance diagnosis through mathematical simulation in table tennis game. Journal of Shanghai University of Sport, 28(2), 68-72.

Zhang, H., Liu, W., Hu, J.J., \& Liu, R.Z. (2013). Evaluation of elite table tennis players' technique effectiveness. Journal of Sports Sciences, 31(14), 1526-1534.

Wu, H., \& Zhang, X. (2002). Training exploration on success of Chinese table tennis team, Beijing: Beijing Sport University.

Wu, X.Z., \& Escobar Vargas, J. (2007a). Notational analysis for competition in table tennis (part I): based format analysis. In X.P. Zhang, D.D. Xiao, \& Y. Dong (Eds.), The proceedings of the Ninth International Table Tennis Federation Sports Science Congress $2^{\text {nd }}$ edition. Paper presented at the $9^{\text {th }}$ ITTF Sports Science Congress, Shanghai, China, 27-30 April 2005 (pp. 104-108). Beijing: People's sports publishing house of China.

Wu, X.Z., \& Escobar Vargas, J. (2007b). Notational analysis for competition in table tennis (part 2): Non-format method. In X.P. Zhang, D.D. Xiao, \& Y. Dong (Eds.), The proceedings of the Ninth International Table Tennis Federation Sports Science Congress $2^{\text {nd }}$ edition. Paper presented at the $9^{\text {th }}$ ITTF Sports Science Congress, Shanghai, China, 27-30 April 2005 (pp. 109-118). Beijing: People's sports publishing house of China.

\section{UTICAJ PROMENA PRAVILA IGRE NA EFIKASNOST U STONOM TENISU: RAZLIKE IZMEĐU POBEDNIKA I PORAŽENIH}

Cilj istraživanja je defnisanje razlika između pobednika i poraženih u stonoteniskim mečevima, $u$ odnosu na promene pravila igre. Analizirani su podaci nakon četiri velike promene pravila igre $u$ 122 meča na uzorku od 244 igrača u periodu od 1996 - 2015. Indikatore igre činili su 30 tehničkih $i$ taktičkih aktivnosti (element igre, ukupan broj $i$ ishod), kao i aktivnosti vezane za servis $i$ vraćanje serve (vrsta, ukupan broj, ishod serve $i$ vraćanja serve). Primenjen je neparametarski MannWhitney-Wilcoxon test $(p \leq 0.05)$. Generalno, broj udaraca u poenu u odnosu na promene pravila bio je promenljiv, uz dominaciju igre forhend udarcima. Rezultati ukazuju na postojanje statistički značajnih razlika između pobednika i poraženih u svim analiziranim periodima: igrajući sa $38 \mathrm{~mm}$ loptom do 21-og poena u osam aktivnosti, igrajući sa $40 \mathrm{~mm}$ loptom do 21-og poena u jednoj aktivnosti, igrajući sa loptom od $40 \mathrm{~mm}$ do 11 poena u sedam aktivnosti $i$ u igranju sa novim oblogama reketa u 14 aktivnosti. Rezultati ukazuju na postojanje različitih modaliteta $i$ stilova igre koji utiču na rezultat i pobedu. Danas igrači koriste različite načine za osvajanje poena, ali se efikasnost servisa i vraćanja serve pokazala kao izuzetno značajan elemnt igre.

Ključne reči: analiza mečeva, individualni sport, sport sa reketom, indikatori učinka 